\title{
Alcances sobre la personalidad y el problema del estrés y las conductas alimentarias de riesgo en la población universitaria no obesa
}

\author{
Effects of personality and the problem of stress and risky \\ eating behaviors in the non-obese university population
}

(iD) Karina Zapata $\square$

Universidad Le Cordon Bleu. Lima, Perú



\section{RESUMEN}

El presente artículo, tiene como objetivo establecer los primeros alcances, a nivel Lima Metropolitana, sobre la problemática del estrés y conductas alimentarias de riesgo y la personalidad en población universitaria no obesa. Una de las etapas más críticas para afianzar estos nuevos hábitos es en la infancia; sin embargo, por los cambios dados a nivel contextual y tecnológico, la etapa universitaria también se ha consolidado como una etapa crítica para el ser humano. Factores como el estrés que de alguna manera son aplacados por ciertos rasgos de personalidad y por los hábitos adquiridos en la infancia; sin embargo, es importante poder establecer un estudio donde se puedan identificar más factores que intervienen en esta problemática y a su vez, hallar métodos de prevención a fin de afianzar hábitos alimenticios saludables en los estudiantes.

Palabras clave: Personalidad, estrés, conductas alimentarias de riesgo.

\begin{abstract}
The aim of this article is to establish the first findings, at Metropolitan Lima level, about the problem of stress and risky eating behaviors and personality in the non-obese university population. One of the most critical stages to reinforce these new habits is during childhood; however, due to the changes that are taking place at contextual and technological level, the university stage is also considered as a critical stage for the human being. Issues such as stress are somehow soothed by certain personality features and by habits acquired during childhood. However, it is important to be able to set up a study to identify more factors that intervene in this problem and, in turn, find prevention methods in order to foster healthy eating behaviors in the students.
\end{abstract}

Keywords: Personality, stress, risky eating behaviors. 


\section{INTRODUCCIÓN}

El estudio de la personalidad se encuentra ligado directamente a aspectos conductuales, sentimientos y pensamientos que en cierta medida influyen en muchos de los comportamientos que ejercen las personas en determinadas situaciones relacionadas a diversos factores. Uno de estos factores es el estrés, que a nivel mundial arroja el 50 \% de relación con algún tipo de enfermedad mental (Caldera y Pulido, 2007). En ese sentido, este se ha convertido en un factor de riesgo para diversas enfermedades, como pueden ser las enfermedades cardiovasculares, entre otras. (Ávila et al., 2014). En un mundo regido por la alta demanda de puestos laborales, los nuevos estudiantes, adultos jóvenes, que están siendo parte del mundo universitario, es importante poder identificar factores que influyen en el desarrollo de capacidades y nuevos aprendizajes $y$, al mismo tiempo, poder controlar los efectos que puedan estar repercutiendo en su personalidad, pues según estudios, Espinoza, Rodríguez, Gálvez, Mac Millan (2011), los rasgos de personalidad guardan relación con diversas áreas de la salud.

Se han encontrado diversos trabajos de investigación que se enfocan en el impacto que tiene el estrés en la ingesta de alimentos en estudiantes universitarios, ya que se encuentran en un periodo de adaptación a los cambios y a la adquisición de nuevos hábitos que pueden provocar problemas a corto o largo plazo en la salud física, mental y también en su desempeño a lo largo de la carrera. En ese sentido, se han venido planteando nuevos mecanismos que ayuden a controlar o mitigar los efectos negativos mencionados. Una de esas propuestas es la intervención de las Funciones Ejecutivas (FE), ya que estas tienen una capacidad regulatoria sobe determinados tipos de conductas de riesgo y a esto se le suma la intervención de factores de personalidad que también ayudan a controlarlas.

Existen estudios en los cuales se observa que las FE tienen la capacidad de reducir el impacto del estrés, ya que ayuda a disminuir la percepción de situaciones amenazantes de acuerdo con el contexto en el que se encuentra el individuo. Asimismo, se han hallado diversos estudios donde se observa que existe diferencias entre los efectos que del estrés en la conducta (Gonzáles, 2018). Esto nos lleva a preguntarnos sobre cuáles serían los factores que intervendrían en los individuos para que los efectos del estrés sean menos impactantes en un grupo de personas más que en otros.

El individuo pasa por diversas etapas en las que sufre cambios a nivel físico y emocional, lo que significa que la falta de prevención ante estas situaciones puede provocar problemas en su salud, de ello la importancia de abarcar factores como estilos de afrontamiento e ingesta de alimentos en grupo que es de especial interés para la psicología de la salud (Chau y Saravia, 2014).

Estudios epidemiológicos de la salud mental en población universitaria presentan alta prevalencia en trastornos depresivos y ansiosos, manteniéndose los trastornos depresivos a lo largo de la carrera entre otros padecimientos como cambios en su ritmo de sueño, tensión, inquietud, cansancio, aburrimiento, etc. (Chau y Saravia, 2014). Junto con ello, el análisis de las de variables como la personalidad y la capacidad regulatoria de las funciones ejecutivas es de suma importancia por su relevancia en la capacidad del individuo al momento de presentarse situaciones estresantes como 
los cambios ocurridos en el camino de su vida universitaria, en especial, las FE que permite al individuo adaptarse cognitiva, emocional y socialmente (Da Silva, 2017).

Las investigaciones publicadas hasta el momento sobre el estudio de estas variables han presentados relaciones que no han sido profundizadas $y$, por ende, presentan deficiencias por la falta de un modelo teórico determinado que respalde los estudios hasta el momento, publicaciones. Por ello, establecer un modelo teórico que ayude a probar las relaciones entre las variables propuestas sería un aporte a nivel teórico. Por otro lado, a nivel social, permitirá una adecuada evaluación neuropsicológica y psicológica en adultos jóvenes quienes, en la actualidad, pertenecen a una generación que se encuentra muy vulnerable a los cambios y que luchan por adaptarse a ellos que es clave en el desarrollo de ciertos trastornos mentales relacionados a las variables estudiadas.

En conclusión, en este artículo se pretende brindar los primeros alcances sobre la problemática expuesta a fin de dar a conocer los factores que pueden servir de ayuda para prevenir las conductas alimentarias de riesgo.

\section{Conducta alimentaria preclínica o de riesgo}

En la actualidad, tanto hombres como mujeres viven en un medio social muy agitado, especialmente en relación con aspecto estético, sin embargo, al encontrarse inmersos en un escenario lleno de exigencias en todos los niveles como el cultural y el social, un gran número modifica hábitos de manera consciente e inconsciente, lo cual hace que pueda incurrir en conductas que son perjudiciales para su bienestar físico y mental. Estas pueden ser conductas en relación con la ingesta de alimentos excesiva o mínima, dependiendo del momento y el grado de ansiedad que se maneje. Por ello, de acuerdo con Unikel, Díaz de León y Rivera (2017), las conductas alimentarias de riesgo son aquellas que rosan a los trastornos de conducta alimentaria (TCA) y pueden ser similares; sin embargo, no son las mismas, ya que, estas son conductas inapropiadas como el uso de medicamentos laxantes, seguimientos de dietas estrictas y restrictivas que sin bien no llegan a tener un diagnóstico clínico, sí son consideradas signos de alerta que requieren de estrategias de prevención a fin de aplacar e impedir algún tipo de daño en niveles superiores. Estas conductas sí pueden llegar a repercutir en la salud de los sujetos, desconociéndose aún si son condicionantes como factores de riesgo para TCA, pero repercuten en el funcionamiento psicosocial, elevando el grado de ansiedad y estrés como lo haría un trastorno alimentario en sí mismo. Según Sánchez (2009), es importante establecer la diferenciación un factor de riesgo y un trastorno, ya que el primero, sería un signo aislado y el segundo, sería la conjunción de signos y factores asociados a un determinado problema, en nuestro caso, a nivel alimenticio.

\section{Cognición social}

Es pertinente mencionar la cognición social, pues esta guarda relación con el control inhibitorio, la monitorización y la planificación, que son vitales para el manejo y la prevención de factores de riesgo de diversa índole, siendo una de ellas el poseer una conducta alimentaria preclínicas. Asimismo, guarda relación con el factor socioambiental, muy estudiado en estos temas.

La cognición social se define como el conjunto de operaciones mentales que subyacen las interacciones sociales, que incluyen la habilidad y capacidad humana de percibir 
las intenciones y disposiciones de otros (Penn et al., 1997 citado por Gutiérrez, 2013). Asimismo, se establece como un aspecto especializado de la cognición cuyo fin más importante es la solución de problemas sociales. Sin embargo, en la actualidad este concepto aún no está del todo estructurado, pues se ha utilizado principalmente dentro de la práctica clínica.

Por otro lado, estudios sobre la elaboración de una perspectiva multidimensional que contemplan aspectos cognitivos y afectivos en el proceso empático, a fin de analizar la relación entre la habilidad empática y las funciones ejecutivas, en suma, de las funciones cognoscitivas han funcionado de forma correcta y han brindado más luz acerca del tema. Ello hace referencia a que las investigaciones han arrojado luz a la compresión del modo en que los componentes cognitivo y emocional de la empatía actúan principalmente en el funcionamiento psicopatológico, sin embargo, es fundamental hacer una exploración sobre las relaciones de estos componentes en individuos que no tienen compromiso psicopatológico (Filippetti, López y Ricahud: 2012).

\section{Personalidad}

Eysenck, de acuerdo con Mori (2002) al hablar de personalidad, se refiere a ella como un todo organizado donde confluye el temperamento, el carácter, el intelecto y el físico de un individuo propiamente expuesto a un determinado ambiente. De acuerdo con Mori (2002), la teoría de la personalidad elaborada por Eysenck está constituida por cuatro factores de conducta: cognitivo, conativo, afectivo y el somático, que abarcan la inteligencias, el carácter, el temperamento y la constitución respectivamente. Esta suma de factores está determinada por la herencia y por el ambiente en el cual se encuentra el individuo. Lo que resalta, además, en la propuesta de Eysenck, la personalidad llegue a ser una ciencia, por ello parte de principios ligados a la objetividad de sus planteamientos como es la parte biológica. En ese sentido, la personalidad no es algo que se encuentre ligado solamente a factores externos como son las sociedades y el medioambiente, sino que, según este autor, al mismo tiempo las conductas que ejercen los seres humanos se encuentran sometidas a factores internos y físicos. Esto hace que el punto de vista cambie en torno al tema, pues se deben hallar formas de modificar conductas no solo en favor de reconocer puntos externos que estén influenciando en ella, sino que se debe incentivar el estudio del funcionamiento del ser humano a nivel interno.

Mori (2002), indica que la teoría de la personalidad de Eysenck, se ha centrado en bases biológicas muy definidas, como por ejemplo, que el hombre biológico guarda relación con el sistema nervioso central y con las inhibiciones corticales.

\section{Estrés y desarrollo académico del estudiante universitario}

El estrés académico es uno de los temas más estudiados en los últimos tiempos, no solo desde el punto de vista de la educación, sino también desde la psicología donde se han relacionado con diversos factores como son el bienestar psicológico y físico. La presencia de niveles altos de ansiedad puede ocasionar consecuencias en el estudiante empezando por un deterioro en el desempeño académico, sin embargo, también se han podido identificar efectos negativos en sus relaciones sociales y en la salud, lo cual provoca, a su vez, deserción estudiantil y ausencias repetitivas por problemas de salud. 
Los estudiantes se enfrentan a niveles más altos de competencias y de exigencias para poder llegar a triunfar en un campo académico determinado. En ese sentido, el estrés vendría a ser la respuesta del cuerpo a condiciones extremas que pueden llegar a perturbar el equilibrio emocional del individuo. Sin embargo, cabe resaltar que las consecuencias del estrés no se producen de manera inmediata, sino que estas son producto de largo de sufrir distintos niveles de tensión, episodios de frustración, experiencias desagradables, desorganización, entre otros, que llegan a un límite y que pueden generar niveles de estrés tan elevados que el cuerpo y la mente del individuo no lo puedan resistir. A ello se suman, los factores como las reglas sociales y la familia que son variables que influyen en gran manera en el ser humano, quien manifiesta el deseo de sentirse perteneciente a un grupo y cumplir con las expectativas sociales. Esto nos lleva a mencionar también que dentro de estos factores podemos hallar el deseo de tener una imagen personal adecuada para la sociedad, como, por ejemplo, "estar en forma", "estar a la moda", etc., que pueden afectar la performance académica del estudiante.

En un estudio realizado con estudiantes de enfermería en el año 2010, Caldera y Pulido (2007), se realizó un rastreo para conocer los factores que ocasionaban estrés que fueron, principalmente, la sobrecarga de trabajos que le asignaban los docentes y la preocupación por las evaluaciones. Mientras que, con respecto a las reacciones físicas y psicológicas que presentaban fueron las siguientes: incapacidad de relajarse y de estar tranquilo, aumento de desgano, reacciones comportamentales, disminución de consumo de alimentos, etc.

\section{Personalidad, estrés, y conductas alimenticias de riesgo}

Hasta el momento hemos podido estudiar brevemente y por separado algunos factores que guardan relación con el tema principal. Por tanto, ahora se abordará el tema de manera conjunta a fin de brindar una primera visión de la problemática que encierran estas tres variables: personalidad, estrés y conductas alimentarias de riesgo.

En un estudio realizado en Santiago de Chile, por Mendoza y colaboradores (2010), donde se evaluaron los hábitos alimenticios, se encontró que los estudiantes tienen una mala percepción de la calidad del servicio de la alimentación y a pesar de la existencia de alimentos saludables y no saludables en su centro de estudio, se denota una gran tendencia a consumir alimentos ricos en carbohidratos y lípidos y ello se suma a su muy baja práctica de actividad física. Esto también lo podemos observar en nuestro contexto, pues no solo por la presencia de espacios donde se puedan adquirir alimentos saludables, sino porque siguen carreras que se caracterizan por la enseñanza de prácticas y hábitos saludables, a pesar de ello, los alumnos prefieren consumir comida que saben que les podría causar algún daño en su salud y esto se ha convertido en una problemática, pues ello, podría deberse a la presencia de factores como el estrés que de alguna manera son aplacados por factores como la personalidad y los hábitos adquiridos en la infancia; sin embargo, es importante poder establecer un estudio donde se puedan identificar más factores que intervienen en esta problemática y, a su vez, hallar métodos de prevención y a fin de afianzar hábitos alimenticios saludables en los estudiantes.

De acuerdo con Espinoza y colaboradores (2011), el llevar un estilo de vida saludable se 
relaciona directamente con la prevención y la disminución de enfermedades crónicas no trasmisibles. Si bien es cierto, una de las etapas más críticas para afianzar estos nuevos hábitos es en la infancia; en consecuencia, por los cambios dados a nivel contextual y tecnológico, la etapa universitaria también se ha consolidado como una etapa crítica para el ser humano. Esto se puede observar mucho en estudiantes que migran a un determinado lugar, pues empeoran su estilo de alimentación por la falta de tiempo para preparar y consumir alimentos hechos en casa y tienden a consumir alimentos muy diferentes a los que solían consumir en su infancia.

Según Troncoso y Amaya (2009) Ios estudiantes hacen referencia que sus conductas alimentarias inadecuadas son causadas por la falta de tiempo para almorzar o por la ansiedad que perciben por las evaluaciones que les conllevan a aumentar su ingesta de alimentos ricos en grasas saturadas, azúcar, etc. Asimismo, hacen hincapié en que sus horarios son muy complicados, pues apenas les da tiempo para comer y hasta muchas veces simplemente no han comido nada en todo el día.

Espinosa, Contreras y Esguerra (2009), establecen que existen diferentes aspectos que ayudan a que se pueda afrontar el estrés, entre ellos, tenemos a los estados optimistas que surgen de la personalidad, pues su buen manejo favorece a la salud mental y la percepción de bienestar y la calidad de vida. Mencionan también que se ha hallado evidencia de que el afrontamiento al estrés o a situaciones estresantes puede estar supeditada de forma individual en relación a factores de la personalidad que intervendrían en el sujeto. En ese sentido, de la teoría psicobiológica de la persona- lidad se distinguen dos componentes: el temperamento y el carácter. Ambos guardan relación con aquellos que se plantea en este artículo, pues, según Espinosa y colaboradores (2009), con el primero se establece que existen sistemas cerebrales independientes para el "mantenimiento e inhibición de la conducta en respuesta a una clase específica de estímulos". Con el carácter se alude al aspecto sociocultural el cual abarca el concepto de sí mismo, sobre los demás y el universo que confluyen sobre lo experimentado y dan como resultado las reacciones emocionales. Por ello, consideramos que ambos términos se encuentran ligados al poder de control, a nivel físico y a nivel emocional de lo nos rodea o suscita en nuestro espacio-tiempo.

\section{CONCLUSIONES}

La personalidad juega un papel importante en el nivel de influencia del estrés en nuestra mente y en nuestro cuerpo y que se debe seguir profundizando estudios relacionados para lograr elaborar métodos de prevención en personas que no presenten métodos para contrarrestar los cambios que pueden repercutir en su salud y bienestar psicológico.

\section{REFERENCIAS BIBLIOGRÁFICAS}

Chau, C. y Saravia, C. (2014). Conductas de salud en Estudiantes Universitarios: Validación del CEVJU. Recuperado de https:// www.aidep.org/sites/default/files/articles/ R41/Art8.pdf

Caldera, F. y Pulido B. (2007). Niveles de estrés y rendimiento académico en estudiantes de la carrera de Psicología del Centro Universitario de los Altos. Revista de Educación y desarrollo. Recuperado de http:// www.cucs.udg.mx/revistas/edu_desarrollo/ anteriores/7/007_Caldera.pdf 
Condori, L. (2013). Relación de: Autoeficacia ante el estrés, personalidad, percepción del bienestar psicológico y de la salud con el rendimiento académico en universitarios (Tesis doctoral). Universidad Nacional Mayor de San Marcos, Perú. Recuperado de http://cybertesis.unmsm.edu.pe/bitstream/handle/cybertesis/3291/Condori_ i|\%282\%29.pdf?sequence $=1$

Da Silva, D. (2017). El estudio de las funciones ejecutivas en una población colombiana de niños y niñas de 7 a 11 años: su valor predictivo en el rendimiento escolar (Tesis doctoral). Universidad Autónoma de Barcelona, España. Recuperado de https://www.tdx.cat/bitstream/handle/10803/461298/dfdsm1de1.pdf?sequence $=1 \&$ is Allowed $=y$

Espinosa, J., Contreras, F., Esguerra, G. (2009). Afrontamiento al estrés y modelo psicobiológico de la personalidad en estudiantes universitarios. Recuperado de https:// www.redalyc.org/pdf/679/67916259008. pdf

Espinoza, L., Rodríguez, F., Gálvez, J., MacMillan, N. (2011). Santiago de Chile. Revista chilena de nutrición, 38,4, p. 458-465.

Gonzáles, M. (2018). Rol modelador de las funciones ejecutivas en la relación entre estrés e ingesta alimentaria en estudiantes universitarios (Tesis de doctorado). Pontificia Universidad Católica de Chile, Santiago de Chile. Recuperado de https://repositorio.uc.cl/bitstream/handle/11534/22000/ Tesis\%20Doctoral\%20Mariela\%20Gonz\%C3\%A1lez\%20Tovar.pdf? sequence=1

Filippetti, V. y Richaud, M. (2012). Aproximación Neuropsicológica al Constructo de Empatía: Aspectos Cognitivos y Neuroana- tómicos. Recuperado de file:///C:/Users/ USUARIO/Downloads/Aproximacionneuropsico logicaalconstructodeempatia.pdf

Gutiérrez, K. (2013). Evaluación neuropsicológica de la cognición social en la esquizofrenia. Pensamiento Psicológico. 11, (2), 103-123.

Mendoza, L., Cabrera, E., González, D., Martínez, R., Pérez, J., Saucedo, R. (2010). Factores que ocasionan estrés en Estudiantes Universitarios. México. Revista de enfermería, 4, 3.

Mori, P. (2002). Personalidad, autoconcepto y percepciones del compromiso parental: sus relaciones con el rendimiento académico en alumnos del sexto grado (Tesis de doctorado). Universidad Nacional Mayor de San Marcos, Perú, Lima.

Troncoso, P. y Amaya, J. (2009). Factores sociales en las conductas alimentarias de estudiantes universitarios. Recuperado de https://scielo.conicyt.cl/scielo.php?pi$d=S 0717-75182009000400005 \&$ script $=s-$ ci_arttext

Unikel, C., Díaz de León, C. y Rivera, J. (2017), Conductas alimentarias de riesgo y factores de riesgo asociados: Desarrollo y validación de instrumentos. México: Universidad Autónoma Metropolitana. 